\title{
Current-induced spin polarization for a general two-dimensional electron system
}

\author{
C. M. Wang ${ }^{*}, 1$, H. T. Cui ${ }^{1}$, and Q. Lin ${ }^{2}$ \\ ${ }^{1}$ Department of Physics, Anyang Normal University, Anyang 455000, China \\ ${ }^{2}$ Shanghai University of Engineering Science, 333 Longteng Road, Songjiang, Shanghai 201620, China
}

PACS 71.70.Ej, 72.10.Bg, 72.25.Dc

* Corresponding author: e-mail cmwangsjtu@gmail.com

In this paper, current-induced spin polarization for twodimensional electron gas with a general spin-orbit interaction is investigated. For isotropic energy spectrum, the in-plane current-induced spin polarization is found to be dependent on the electron density for non-linear spin-orbit interaction and increases with the increment of sheet density, in contrast to the case for $k$-linear spin-orbit coupling model. The numerical evaluation is performed for InAs/InSb heterojunction with spin-orbit coupling of both linear and cubic spin-orbit coupling types. For $\delta$-type short-range electron-impurity scatteri- $\mathrm{ng}$, it is found that the current-induced spin polarization increases with increasing the density when cubic spin-orbit couplings are considered. However, for remote disorders, a rapid enhancement of current-induced spin polarization is always observed at high electron density, even in the case without cubic spin-orbit coupling. This result demonstrates the collision-related feature of current-induced spin polarization. The effects of different high order spin-orbit couplings on spin polarization can be comparable.
1 introduction Spintronics, where the spin degree of freedom is manipulated to control the electronic devices, has been becoming a rapid field of condensed matter physics [1]. Current-induced spin polarization (CISP) discloses the possibility of the spin polarization generated in semiconductor directly by the electric field. It refers to a spatially homogeneous spin polarization in two dimensional systems due to an in-plane charge current. CISP due to spin-dependent scattering was first reported by D'yakonov and Perel' in 1971 [2]. Later it was realized that such transport phenomenon exists in semiconductorbased two-dimensional electron gas (2DEG) without structure or bulk inversion symmetry [3, 4, 5]. Experimentally, CISP was first measured by Silov et al. in two dimensional hole system with the help of polarized photoluminescence technology [6]. Observations of CISP in strained semiconductors have been reported by Kato [7,8]. And later Sih et al. demonstrated the existence of CISP in AlGaAs quantum well [9].

So far, the theoretical investigations about the CISP are mainly focused on the 2DEG with $\boldsymbol{k}$-linear SOC, such as
Rashba SOC due to structure inversion asymmetry $H_{R}=$ $\alpha\left(k_{y} \sigma_{x}-k_{x} \sigma_{y}\right)$ [3,5,10], linear Dresselhaus SOC due to bulk inversion asymmetry $H_{D}^{(1)}=\beta\left(k_{x} \sigma_{x}-k_{y} \sigma_{y}\right)$ [11], and the combination of linear Rashba and Dresselhaus coupling types [12, 13, 14]. Here $\boldsymbol{\sigma}=\left(\sigma_{x}, \sigma_{y}, \sigma_{z}\right)$ represents the set of Pauli matrices, and $\boldsymbol{k}=\left(k_{x}, k_{y}\right)=$ $k(\cos \theta, \sin \theta)$ is the two-dimensional momentum, $\alpha$ and $\beta$ are linear Rashba and Dresselhaus SOC factors, respectively. CISP is found to be proportional to the SOC constant and independent of the electron density for 2DEG with $\boldsymbol{k}$-linear Rashba or Dresselhaus SOC when a dc electric field is applied [3,5, 11].

However situations may become different for $2 \mathrm{DEG}$ with non-linear SOC, just like the spin Hall effect [15, 16, 17, 18, 19, 20,21,22], the investigation of CISP on this system is desirable. Liu et al. studied the CISP in holedoped two dimensional system lacking structure inversion symmetry, and they found that CISP is dependent on the Fermi energy, in vivid contrast against the case in $2 \mathrm{DEG}$ with $k$-linear SOC [23]. While it was pointed by Liu et al. that the "spin" for hole system is actually the total an- 
gular momentum, where the spin of hole system is not a conserved physical quantity [23]. Hence, we expect the behavior of the spin polarization of conduction electron in the presence of non-linear SOC. We know that at high electron sheet density, the cubic term of Dresselhaus SOC, $H_{D}^{(3)}=\eta\left(k_{x} k_{y}^{2} \sigma_{x}-k_{x}^{2} k_{y} \sigma_{y}\right)$ [24], ( $\eta$ is the cubic Dresselhaus SOC constant), has to be taken into account [25]. Recently, by using the double-group representations, Cartoixà et al. [26] found that there is another cubic term for heterojunction due to bulk inversion asymmetry, $H_{B I A}^{(3)}=$ $\zeta\left(k_{y}^{3} \sigma_{y}-k_{x}^{3} \sigma_{x}\right)$, (i. $e$. the last term of Eq. (3) in Ref. [26]). Here $\zeta=a^{2} \beta / 6$ is the cubic SOC constant with $a$ as the well width. Apart from the type of SOC, most of the pioneering researches on CISP treat the electronimpurity collision with a simple momentum-independent form, described by a relaxation time $\tau$. However in realistic 2DEG, the electron density is not large enough to screen the charged impurities. The interaction between electron and disorder is long ranged.

In this paper we consider the CISP in 2DEG with a general SOC, which can be applied to describe Rashba, linear and cubic Dresselhaus SOCs, and many other SOCs. For the simple isotropic energy band form, the analytical result of CISP is obtained. The numerical evaluation for the electron system with both linear and cubic SOCs due to bulk inversion asymmetry is also performed, considering both short- and long-range disorders.

2 formalism We consider a two-dimensional noninteracting electron system with a general SOC, described by the following one-particle Hamiltonian:

$$
\hat{H}=\varepsilon_{0}(\boldsymbol{k})+\boldsymbol{b}(\boldsymbol{k}) \cdot \boldsymbol{\sigma} .
$$

For simplicity we have assumed that $\varepsilon_{0}(\boldsymbol{k})$, the energy dispersion in the absence of SOC, is isotropic function of momentum $\boldsymbol{k}$. We set $\hbar=1$ throughout this paper. As a further simplification of SOC, we shall later specialize to the model, where the spin orbit field $\boldsymbol{b}(\boldsymbol{k})$ has the form

$$
b_{x}(\boldsymbol{k})+i b_{y}(\boldsymbol{k})=\tilde{\alpha} k^{M_{1}}\left(\sin M_{2} \theta\right)^{\lambda} e^{i M_{3} \theta} .
$$

Here the complex number $\tilde{\alpha}=\alpha_{r}+i \alpha_{i}$ is the general coupling constant, irrespective of the momentum $k$, with $\alpha_{r}$ and $\alpha_{i}$ as the real and imaginary part, respectively. $\lambda=0,1$ and $M_{1}, M_{2}, M_{3}$ are integer numbers. It will be noted later that the index $\lambda$ determines whether the energy spectrum is isotropic. The number $M_{1}$ usually is positive. It is found that when $\lambda=0$ and $M_{1}=M_{3}$, our model becomes the one in Ref. [21], where the model is used to discuss the spin Hall effect. Due to time reversal symmetry, the spin orbit field $\boldsymbol{b}(\boldsymbol{k})$ satisfies $\boldsymbol{b}(\boldsymbol{k})=-\boldsymbol{b}(-\boldsymbol{k})$. Therefore for the case $\lambda=0, i$. $e$. isotropic energy spectrum, $M_{3}$ must be an odd integer number; while for the case $\lambda=1, i$. e. anisotropic energy spectrum, $M_{2}+M_{3}$ must be an odd integer number. Now we list some special SOC forms: for pure Rashba SOC, $\tilde{\alpha}=-i \alpha, \lambda=0, M_{1}=M_{3}=1$; while for $k$-linear
Dresselhaus SOC, $\tilde{\alpha}=\beta, \lambda=0, M_{1}=1, M_{3}=-1$; and the case $\tilde{\alpha}=-\frac{1}{2} i \eta, \lambda=1, M_{1}=3, M_{2}=2, M_{3}=1$ corresponds the cubic Dresselhaus term.

With the help of the local unitary matrix

$$
U_{\boldsymbol{k}}=\frac{1}{\sqrt{2}}\left(\begin{array}{cc}
1 & 1 \\
i e^{i \chi_{\boldsymbol{k}}} & -i e^{i \chi_{\boldsymbol{k}}}
\end{array}\right) \text {, }
$$

where $\chi_{\boldsymbol{k}}$ satisfies

$$
\tan \chi_{\boldsymbol{k}}=\frac{\alpha_{i} \sin M_{3} \theta-\alpha_{r} \cos M_{3} \theta}{\alpha_{i} \cos M_{3} \theta+\alpha_{r} \sin M_{3} \theta},
$$

the Hamiltonian (1) can be diagonalized into $H=$ $\operatorname{diag}\left[\varepsilon_{1}(\boldsymbol{k}), \varepsilon_{2}(\boldsymbol{k})\right]$ in the helicity basis. Here

$$
\varepsilon_{\mu}(\boldsymbol{k})=\varepsilon_{0}(\boldsymbol{k})+(-1)^{\mu} \varepsilon_{M}(\boldsymbol{k}),
$$

with $\varepsilon_{M}(\boldsymbol{k})=|\tilde{\alpha}| k^{M_{1}}\left(\sin M_{2} \theta\right)^{\lambda}$ and $\mu=1,2$ as the helix band index. We note that when $\lambda=0$, the energy dispersion $\varepsilon_{\mu}(\boldsymbol{k})$ becomes isotropic function of wave vector $\boldsymbol{k}$, whereas for the case $\lambda=1$, the energy spectrum relies on the angle of momentum.

When the system is driven by a weak dc electric field applied along the $\hat{x}$ direction, $\boldsymbol{E}=E_{0} \hat{x}$. Following the procedure of Ref. [27], the kinetic equation of the distribution function $\rho(\boldsymbol{k})$ can be derived, with the equilibrium distribution function

$$
\rho^{(0)}=\left(\begin{array}{cc}
n_{\mathrm{F}}\left[\varepsilon_{1}(\boldsymbol{k})\right] & 0 \\
0 & n_{\mathrm{F}}\left[\varepsilon_{2}(\boldsymbol{k})\right]
\end{array}\right) .
$$

Here $n_{\mathrm{F}}(x)$ is the Fermi distribution. The distribution function $\rho(\boldsymbol{k})$ to first order of electric field comprises two terms. The first term is written as

$\rho_{12}^{(1)}=\rho_{21}^{(1)}=-\frac{e E_{0}}{4 \varepsilon_{M}(\boldsymbol{k})} \frac{\partial \chi_{\boldsymbol{k}}}{\partial k_{x}}\left\{n_{\mathrm{F}}\left[\varepsilon_{1}(\boldsymbol{k})\right]-n_{\mathrm{F}}\left[\varepsilon_{2}(\boldsymbol{k})\right]\right\}$.

And the second term $\rho^{(2)}(\boldsymbol{k})$ is determined by the set of equations with the form

$$
\begin{aligned}
e E_{0} \frac{\partial n_{\mathrm{F}}\left[\varepsilon_{\mu}(\boldsymbol{k})\right]}{\partial k_{x}} & =\pi \sum_{\boldsymbol{q} \mu^{\prime}}|u(\boldsymbol{k}-\boldsymbol{q})|^{2} \Omega_{\mu \mu^{\prime}} \\
\times & {\left[\rho_{\mu \mu}^{(2)}(\boldsymbol{k})-\rho_{\mu^{\prime} \mu^{\prime}}^{(2)}(\boldsymbol{q})\right] \delta\left(\varepsilon_{\mu}(\boldsymbol{k})-\varepsilon_{\mu^{\prime}}(\boldsymbol{q})\right), } \\
4 \varepsilon_{M}(\boldsymbol{k}) \operatorname{Re} \rho_{12}^{(2)}(\boldsymbol{k}) & =\pi \sum_{\boldsymbol{q} \mu \mu^{\prime}}|u(\boldsymbol{k}-\boldsymbol{q})|^{2} \bar{\Omega}_{\mu \mu^{\prime}} \\
\times & {\left[\rho_{\mu \mu}^{(2)}(\boldsymbol{k})-\rho_{\mu^{\prime} \mu^{\prime}}^{(2)}(\boldsymbol{q})\right] \delta\left(\varepsilon_{\mu}(\boldsymbol{k})-\varepsilon_{\mu^{\prime}}(\boldsymbol{q})\right) . }
\end{aligned}
$$

Here $\Omega_{\mu \mu^{\prime}}=1+(-1)^{\mu+\mu^{\prime}} \cos \left(\chi_{\boldsymbol{k}}-\chi_{\boldsymbol{q}}\right)$ and $\bar{\Omega}_{\mu \mu^{\prime}}=$ $(-1)^{\mu^{\prime}} \sin \left(\chi_{\boldsymbol{k}}-\chi_{\boldsymbol{q}}\right) . \operatorname{Re} \rho_{12}^{(2)}(\boldsymbol{k})$ represents the real part 
of the off-diagonal distribution function $\rho_{12}^{(2)}(\boldsymbol{k}) . u(\boldsymbol{k})$ is the electron-impurity scattering matrix. Note that the above equations of distribution function, Eqs. (8) and (9), have been derived in Refs. [3, 4, 13].

Finally, in helix spin basis, the single-particle operators of spin polarization are given by $\hat{S}_{i}=U_{\boldsymbol{k}}^{\dagger} \frac{1}{2} \sigma_{i} U_{\boldsymbol{k}}$ with $i=x, y, z$. The corresponding macroscopical quantities are obtained by taking the statistical average over them, $S_{i}=\sum_{\boldsymbol{k}} \operatorname{Tr}\left[\rho(\boldsymbol{k}) \hat{S}_{i}\right]$, and are expressed as

$$
\begin{aligned}
S_{x} & =\frac{1}{2} \sum_{\boldsymbol{k} \mu} \sin \chi_{\boldsymbol{k}}\left[\rho_{22}(\boldsymbol{k})-\rho_{11}(\boldsymbol{k})\right], \\
S_{y} & =\frac{1}{2} \sum_{\boldsymbol{k} \mu} \cos \chi_{\boldsymbol{k}}\left[\rho_{22}(\boldsymbol{k})-\rho_{11}(\boldsymbol{k})\right], \\
S_{z} & =\sum_{\boldsymbol{k}} \operatorname{Re} \rho_{12}(\boldsymbol{k}) .
\end{aligned}
$$

\section{3 spin polarization}

3.1 analytical result For this angle-dependent SOC, the formulas to be derived will become much less transparent, and the integrals are more difficult to solve analytically. Therefore, we first limit ourselves to isotropic energy spectrum, $i$. e. $\lambda=0$ and the parabolic case $\varepsilon_{0}(\boldsymbol{k})=\frac{k^{2}}{2 m}$. The spin polarization is examined in the presence of electron-impurity scattering with $\delta$-potential, $|u(\boldsymbol{k}-\boldsymbol{q})|^{2}=n_{i} u_{0}^{2}$. Here $m$ is the effective mass of two-dimensional electrons and $n_{i}$ is the impurity density.

Keeping only the lowest-order of spin-orbit interaction, the diagonal elements of distribution function can be obtained analytically from Eq. (8). For $M_{3}= \pm 1$, the diagonal elements of $\rho^{(2)}(\boldsymbol{k})$ take the form

$$
\begin{aligned}
\rho_{11}^{(2)}(\boldsymbol{k})= & -\frac{e E_{0} \tau}{m}\left[k+m|\tilde{\alpha}|\left(1-M_{1}\right)(2 \pi N)^{\frac{M_{1}-1}{2}}\right] \\
& \times \cos \theta \delta\left(\varepsilon_{\boldsymbol{k} 1}-\varepsilon_{F}\right), \\
\rho_{22}^{(2)}(\boldsymbol{k})= & -\frac{e E_{0} \tau}{m}\left[k-m|\tilde{\alpha}|\left(1-M_{1}\right)(2 \pi N)^{\frac{M_{1}-1}{2}}\right] \\
& \times \cos \theta \delta\left(\varepsilon_{\boldsymbol{k} 2}-\varepsilon_{F}\right) .
\end{aligned}
$$

And for the case $M_{3}>1$ or $M_{3}<-1$, they are given by

$$
\begin{aligned}
& \rho_{11}^{(2)}(\boldsymbol{k})=-e E_{0} \tau\left|\frac{\partial \varepsilon_{1}(\boldsymbol{k})}{\partial k}\right| \cos \theta \delta\left(\varepsilon_{\boldsymbol{k} 1}-\varepsilon_{F}\right), \\
& \rho_{22}^{(2)}(\boldsymbol{k})=-e E_{0} \tau\left|\frac{\partial \varepsilon_{2}(\boldsymbol{k})}{\partial k}\right| \cos \theta \delta\left(\varepsilon_{\boldsymbol{k} 2}-\varepsilon_{F}\right),
\end{aligned}
$$

with $\tau=1 / m n_{i} u_{0}^{2}$ as the relaxation time, $\varepsilon_{F}$ as the Fermi energy. With the help of Eq. (9), the off-diagonal distribution function can be obtained directly. Finally, we find the spin polarization takes the form:

$S_{x}=\left\{\begin{array}{ll}\frac{e m \alpha_{r} \tau E_{0}}{2 \pi}(2 \pi N)^{\frac{M_{1}-1}{2}}, & \left|M_{3}\right|=1 \\ 0, & \left|M_{3}\right|>1\end{array}\right.$,
$S_{y}=\left\{\begin{array}{ll}\frac{e m \alpha_{i} \tau E_{0}}{2 \pi}(2 \pi N)^{\frac{M_{1}-1}{2}}, & \left|M_{3}\right|=1 \\ 0, & \left|M_{3}\right|>1\end{array}\right.$,

$S_{z}=0$.

We come to the conclusion that the in-plane CISPs exist only when the winding number $\left|M_{3}\right|=1$, nevertheless the out-of-plane component of CISP is always zero for this 2DEG with isotropic general SOC. As expected, for $M_{3}= \pm 1$ and $M_{1}=1$, we obtain the CISP for $2 \mathrm{DEG}$ with Rashba or $\boldsymbol{k}$-linear Dresselhaus SOC, in agreement with previous theoretical studies [3, 5, 11, 12, 13]. Our results imply that, in contrast to the 2DEG with Rashba or $\boldsymbol{k}$ linear Dresselhaus SOC, the in-plane CISPs for non-linear SOC system, $M_{1}>1$, become dependent on the electron density and enhance for high density semiconductor. The above analytical calculation is valid in the weak SOC case, $\varepsilon_{M}\left(k_{\mathrm{F}}\right) \ll \varepsilon_{0}\left(k_{\mathrm{F}}\right)$, but the relationship between $\varepsilon_{M}\left(k_{\mathrm{F}}\right)$ and $\tau^{-1}$ is arbitrary. Here $k_{\mathrm{F}}$ is the Fermi wave vector. For strong SOC case, the dependence of CISP on the electron density can be evaluated numerically.

It should be noted that the in-plane CISP comes from the interband processes, arising from the SOC, which can be seen from Eqs. 10 and (11). In the absence of spinorbit interaction, $\rho_{11}=\rho_{22}$ leads to the vanishing CISP. Hence, although the result, we obtained here, is for the parabolic case, the nonparabolic contribution of $\varepsilon_{0}(\boldsymbol{k})$ to CISP may only change its value slightly through the Fermi energy. Further, it can be confirmed below by the numerical calculation. In general, SOC field will be the combination of Eq. 22 with both linear and high order terms. From our analytical result, one can deduce that with increasing the high order SOC constant, the density-related feature of CISP will become more and more evident.

3.2 numerical result Now we perform the numerical evaluation for CISP in InAs/InSb heterojunctions without the additional large bias voltage, where the main SOC contribution terms arise owing to the absence of the center of inversion in the bulk material.

Further, to take account of the nonparabolicity of the energy band of InAs, we use the isotropic Kane band model:

$$
\varepsilon_{0}(k)=\frac{1}{2 \gamma}\left(\sqrt{1+2 \gamma \frac{k^{2}}{m}}-1\right),
$$

where $\gamma \approx 1 / \varepsilon_{g}$ is the nonparabolic parameter, with $\varepsilon_{g}$ as the energy gap between the conduction and valence bands. Note that Kane energy band becomes the parabolic case for vanishing $\gamma$. The nonparabolic factor in the numerical calculation is set to be $\gamma=2.73 \mathrm{eV}^{-1}$ for InAs [28]. The 


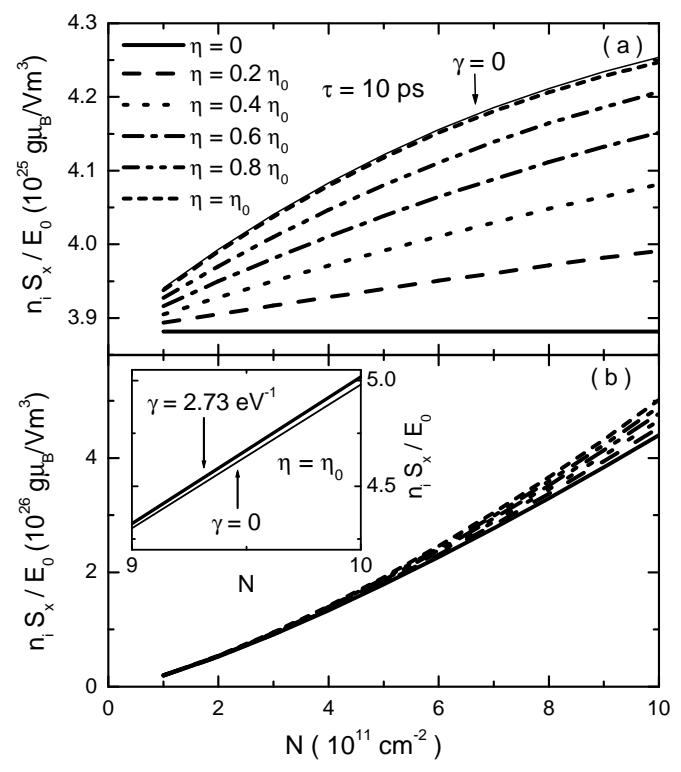

Figure $1 n_{i} S_{x} / E_{0}$ as functions of electron density for (a) short- or (b) long-range impurity scattering for $\gamma=$ $2.73 \mathrm{eV}^{-1}$. Here $\eta_{0}=1.0 \times 10^{-28} \mathrm{eVm}^{3}, g$ is the effective $\mathrm{g}$-factor, and $\mu_{B}$ is the Bohr magneton. The thin solid line in (a) is obtained for $\gamma=0$ and $\eta=\eta_{0}$. The thin and thick solid lines in inset of (b) is calculated when $\eta=\eta_{0}$ for $\gamma=0$ and $\gamma=2.73 \mathrm{eV}^{-1}$, respectively. The unit of electron density $N$ is $10^{11} \mathrm{~cm}^{-2}$, and the unit of $n_{i} S_{x} / E_{0}$ is $10^{26} \mathrm{~g} \mu_{\mathrm{B}} / \mathrm{Vm}^{3}$.

$\delta$-form short-range or the remote charged impurity scattering is considered in the calculation. The scattering matrix of remote electron-impurity scattering takes the form: $|u(q)|^{2} \simeq n_{i} e^{-2 s q} I(q)^{2}$ [29], with $I(q)$ as the form factor. We set the electron effective mass at the band bottom $m=0.04 m_{\mathrm{e}}\left(m_{\mathrm{e}}\right.$ is the free electron mass), remote impurities in InSb barrier are located at a distance of $s=10 \mathrm{~nm}$ from the interface of the heterojuction [22,28].

3.2.1 $\boldsymbol{H}_{D}^{(1)}+\boldsymbol{H}_{D}^{(3)}$ First we consider the system with linear and cubic Dresselhaus SOC. In this case, the spin orbit field $\boldsymbol{b}(\boldsymbol{k})=\left(\beta k_{x}+\eta k_{x} k_{y}^{2},-\beta k_{y}-\eta k_{y} k_{x}^{2}\right)$. At the same time, the equations about distribution function, from Eq. (6) to Eq. 9), can be obtained, by substituting the new form of energy $\varepsilon_{M}(\boldsymbol{k})$ and $\chi_{\boldsymbol{k}}$ with $\varepsilon_{M}(\boldsymbol{k})=$ $\sqrt{b_{x}(\boldsymbol{k})^{2}+b_{y}(\boldsymbol{k})^{2}}, \chi_{\boldsymbol{k}}=-\tan ^{-1} \frac{b_{x}(\boldsymbol{k})}{b_{y}(\boldsymbol{k})}$. It is noted that the energy spectrum becomes anisotropic completely.

From Eqs. 8 and (10), we find that the $x$ component of CISP is inverse proportional to the impurity density $n_{i}$. Therefore, $n_{i} S_{x} / E_{0}$ is plotted in these figures. In the calculation, we set linear Dresselhaus SOC coefficient $\beta=1.0 \times 10^{-11} \mathrm{eVm}$. The short-range impurity scattering is considered with relaxation time $\tau=10 \mathrm{ps}$. When the cubic Dresselhaus SOC is considered, it is found that CISP is still along the $x$ direction.

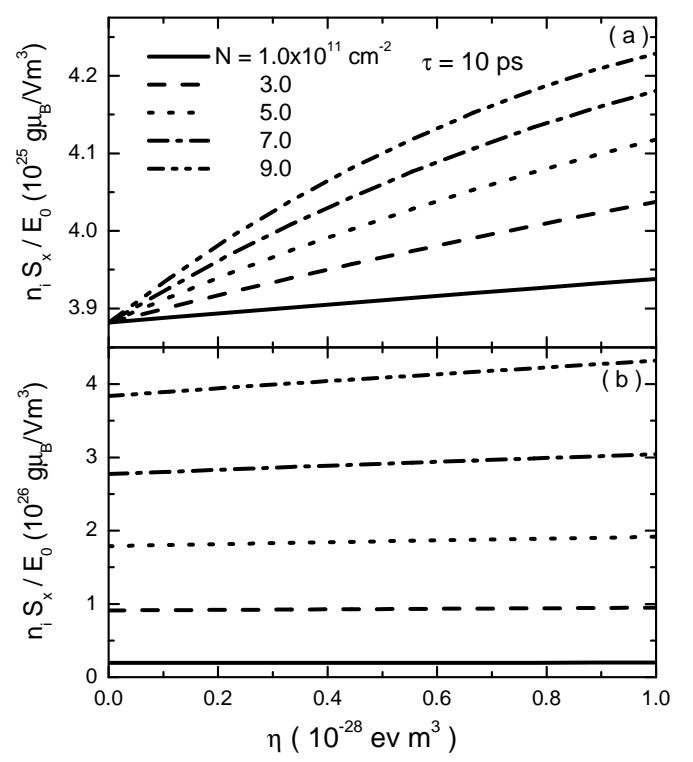

Figure 2 Dependencies of $n_{i} S_{x} / E_{0}$ on cubic Dresselhaus SOC parameter for (a) short- or (b) long-range impurity scattering.

In Fig. 1, the $x$ component of CISP is plotted as function of electron density. For short-range disorder, the density dependence of CISP can be observed when $\eta \neq 0$. Note that the resultant $S_{x}$ is proportional to relaxation time $\tau$. With the increment of the sheet density, CISP increases monotonously, and almost saturates at high density for large $\eta$. From Fig. 1 (b), for long-range electron-impurity scattering it is evident that, unlike the case for short-range disorder, here CISP always increases with ascending the density even for the system without cubic SOC. In the parameter regime, $N<10^{12} \mathrm{~cm}^{-2}$, long-range disorders have strong effect on CISP, where CISP increases rapidly with the rise of density. It can be seen that the role of cubic term of Dresselhaus SOC on CISP becomes important at high sheet density for both short- and long-range collision. CISP for parabolic energy band is also plotted in this figure with a thin line. We find that the weak effect of nonparabolicity on CISP appears at high density.

CISP is shown as a function of the cubic Dresselhaus SOC parameter $\eta$ in Fig. 2. For momentum independent potential, CISP begins with the value $e m \beta \tau E_{0} / 2 \pi$, independent of the density, and increases with ascending $\eta$. In Fig. 2(b), the calculated CISP for long-range collision is almost linear proportional to cubic Dresselhaus constant $\eta$.

3.2.2 $H_{D}^{(1)}+H_{D}^{(3)}+H_{B I A}^{(3)}$ In this subsection, the additional high-order contribution $H_{B I A}^{(3)}$ due to bulk inversion asymmetry in Ref. [26] is also considered. Now the spin orbit field becomes $\boldsymbol{b}(\boldsymbol{k})=\left(\beta k_{x}+\eta k_{x} k_{y}^{2}-\right.$ $\left.\zeta k_{x}^{3},-\beta k_{y}-\eta k_{y} k_{x}^{2}+\zeta k_{y}^{3}\right)$, and the corresponding $\varepsilon_{M}(\boldsymbol{k})$, $\chi_{\boldsymbol{k}}$ can be obtained analogously. We take the well width 


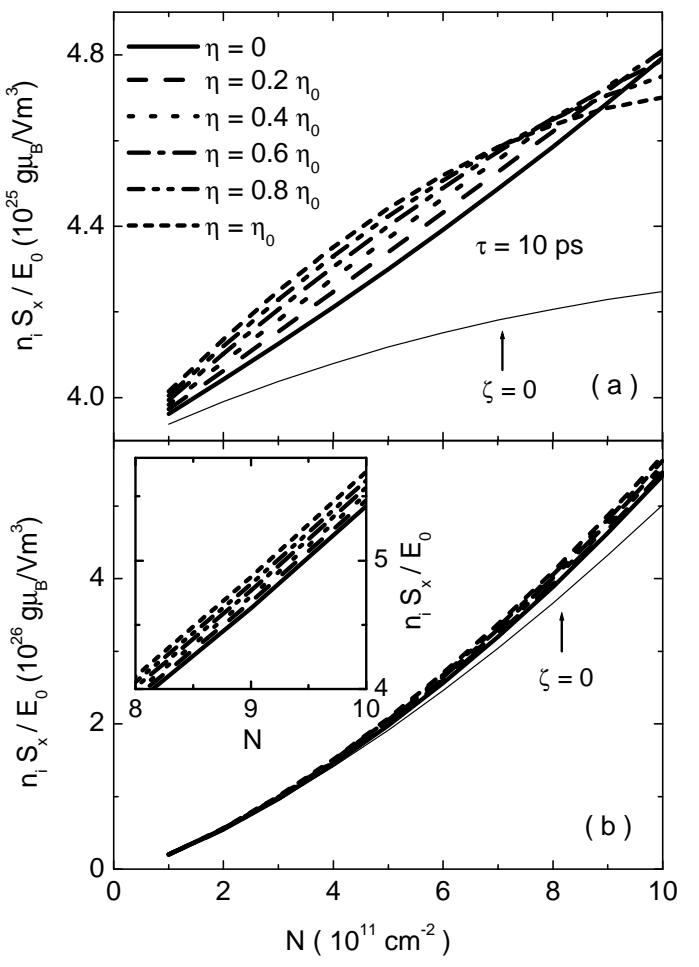

Figure $3 n_{i} S_{x} / E_{0}$ is shown as functions of electron density for (a) short- or (b) long-range impurity scattering when the additional cubic SOC term $H_{B I A}^{(3)}$ is considered. The thin solid lines in (a) and (b) are obtained when $\zeta=0$ and $\eta=\eta_{0}$ for short-range and long-range collisions, respectively. The inset in (b) shows the dependencies of $n_{i} S_{x} / E_{0}$ on $N$ at high density regime. The other parameters are the same as those in Figure 1

$a=5 \mathrm{~nm}$. The calculated $n_{i} S_{x} / E_{0}$ as functions of electron density $N$ is shown in Fig. 3 .

When the additional high-order term $H_{B I A}^{(3)}$ is included, the magnitude of CISP rises for both short- and long-range disorders. For short-range scattering, $n_{i} S_{x} / E_{0}$ always increases with ascending the density. However, at high density, the magnitude of CISP for large $\eta$ may be less than the one for small $\eta$. This is due to the interplay between two cubic terms. It has been seen that CISP saturates at high density when only the Dresselhaus cubic term $H_{D}^{(3)}$ is included. However, one can see, from the thick solid line in Fig. 3(a), this behavior will not occur when we only include the term $H_{B I A}^{(3)}$. For large $\eta$, the effect on CISP of cubic Dresselhaus term $H_{D}^{(3)}$ exceeds the one of this additional cubic term $H_{B I A}^{(3)}$. CISP saturates again at high density, hence its magnitude becomes less than the one with small $\eta$. However, such phenomenon can not be observed for the case of long-range collision.
4 conclusion In summary, the CISP for 2DEG with a general SOC is investigated. For isotropic energy band, we find that the in-plane CISP becomes density-dependent for non-linear SOC, and increases with enhancing the sheet density. We have numerically studied the linear and cubic SOC contributions to CISP, considering both the short- and long-range disorders. For short-range collision, we have demonstrated the dependencies of CISP on density when high-order SOCs are included. When impurity scattering becomes long ranged, however, CISP increases rapidly with raising the density even for the system without cubic SOC. Our investigation indicates that the remote disorder has a strong influence on spin polarization, and the magnitude of CISP strongly relies on the scattering matrix. The contributions of different cubic SOCs to CISP can be comparable.

Acknowledgements We are very thankful to N. S. Averkiev for useful information. HTC gratefully acknowledges support from the Special Foundation of Theoretical Physics of NSF in China (grant 10747159).

\section{References}

[1] I. Žutić, J. Fabian, and S. D. Sarma, Rev. Mod. Phys. 76, 323 (2004).

[2] M. I. D’yakonov and V.I. Perel', Phys. Lett. A 35, 459 (1971).

[3] V. M. Edelstein, Solid State Commun. 73, 233 (1990).

[4] A. G. Aronov, Y. B. Lyanda-Geller, and G. E. Pikus, Sov. Phys. JETP 73, 537 (1991).

[5] J. I. Inoue, G. E. W. Bauer, and L. W. Molenkamp, Phys. Rev. B 67, 033104 (2003).

[6] A. Y. Silov, P.A. Blajnov, J.H. Wolter, R. Hey, K. H. Ploog, and N.S. Averkiev, Appl. Phys. Lett. 85, 5929 (2004).

[7] Y. K. Kato, R. C. Myers, A. C. Gossard, and D. D. Awschalom, Nature (London) 427, 50 (2004).

[8] Y. K. Kato, R. C. Myers, A. C. Gossard, and D. D. Awschalom, Phys. Rev. Lett. 93, 176601 (2004).

[9] V. Sih, R. C. Myers, Y. K. Kato, W. H. Lau, A. C. Gossard, and D. D. Awschalom, Nature Physics 1, 31 (2005).

[10] V. V. Bryksin and P. Kleinert, Phys. Rev. B 73, 165313 (2006).

[11] M. Trushin and J. Schliemann, Phys. Rev. B 75, 155323 (2007).

[12] A. V. Chaplik, M. V. Entin, and L. I. Magarill, Physica E 13, 744 (2002).

[13] N. S. Averkiev, and A. Yu. Silov, Semiconductors 39, 1323 (2005).

[14] V. V. Bryksin and P. Kleinert, Int. J. Mod. Phys. B 20, 1 (2006).

[15] J. E. Hirsch, Phys. Rev. Lett. 83, 1834 (1999).

[16] S. Murakami, N. Nagaosa, and S.-C. Zhang, Science 301, 1348 (2003).

[17] J. Sinova, D. Culcer, Q. Niu, N. Sinitsyn, T. Jungwirth, and A. MacDonald, Phys. Rev. Lett. 92, 126603 (2004).

[18] B. A. Bernevig and S.C. Zhang, Phys. Rev. Lett. 95, 016801 (2005). 
[19] A. G. Mal'shukov and K. A. Chao, Phys. Rev. B 71, 121308 (2005).

[20] S. Murakami, Phys. Rev. B 69, 241202 (2004).

[21] A. V. Shytov, E. G. Mishchenko, H. A. Engel, and B. I. Halperin, Phys. Rev. B 73, 075316 (2006).

[22] Q. Lin, S. Y. Liu, and X. L. Lei, Appl. Phys. Lett. 88, 122105 (2006).

[23] C. X. Liu, B. Zhou, S. Q. Shen, and B. fen Zhu, Phys. Rev. B 77, 125345 (2008).

[24] G. Dresselhaus, Phys. Rev. 100, 580 (1955).

[25] B. Jusserand, D. Richards, H. Peric, and B. Etienne, Phys. Rev. Lett. 69, 848 (1992).

[26] X. Cartoixà, L.-W. Wang, D.Z.-Y. Ting, and Y.-C.Chang, Phys. Rev. B 73, 205341 (2006).

[27] S. Y. Liu and X. L. Lei, Phys. Rev. B 72, 155314 (2005).

[28] J. C. Cao, X. L. Lei, A. Z. Li, M. Qi, and H. C. Liu, Semicond. Sci. Technol. 17, 215 (2002).

[29] X. L. Lei, J. L. Birman, and C. S. Ting, J. Appl. Phys. 58, 2270 (1985). 